\section{Ketamine meets mTOR}

\section{By Tim Fulmer, Senior Writer}

A team from Yale School of Medicine has found an mTOR-mediated mechanism that could help explain how the anesthetic ketamine is able to reduce depression more rapidly than standard antidepressants. ${ }^{1}$ The researchers now hope to identify new targets in the mTOR pathway that can produce the same rapid result without the psychotic side effects associated with ketamine.

Ketamine is marketed as a veterinary anesthetic and as an analgesic to treat severe or chronic pain in humans. At high doses, the drug can cause hallucinogenic and psychotic effects. As a result, ketamine is a recreational drug of abuse and was classified as a Schedule III controlled substance by the U.S. Drug Enforcement Agency (DEA) in 1999.

Ketamine antagonizes the NMDA receptor (NMDAR), which is expressed throughout the CNS and binds the neurotransmitter glutamate on inhibitory neurons. By doing this, the drug is thought to increase the overall excitatory activity of certain brain regions. This heightened activity is a double-edged sword: it can lead to schizophrenia-like cognitive and behavioral abnormalities in animals and normal human subjects ${ }^{2,3}$ but it can also reduce depression in both animals and patients ${ }^{4,5}$ (see Box 1, "NOX2-driven schizophrenia").

In pain, injury to the CNS leads to increased extracellular glutamate, which activates NMDAR on postsynaptic neurons, leading to greater intracellular calcium, more neuronal firing and the development of chronic and/or neuropathic pain. In this context, blockade of NMDAR results in anesthesia. ${ }^{6}$

In 2000 and 2006, groups at Yale and the NIH's National Institute of Mental Health (NIMH) separately published data from small placebocontrolled trials showing that single doses of ketamine relieved depression in a significant number of treatment-resistant patients. The effects were rapid-often within two hours-with relief lasting several days., ${ }^{7,8}$ Most standard antidepressants take about two weeks or longer before a treatment effect is seen.

A team led by Yale's Ronald Duman, professor of psychiatry and pharmacology and director of the Division of Molecular Psychiatry and Abraham Ribicoff Research Facilities, turned to rodents to get a better idea of the cellular signaling pathways that might underlie ketamine's antidepressant properties.
The researchers focused on pathways known to influence synaptic plasticity and connectivity, which are important for learning and memory and are often impaired in the prefrontal cortex and hippocampus of depressed patients. ${ }^{9,10}$

They found that mammalian target of rapamycin (mTOR; FRAP; RAFT1), a protein complex known to be involved in protein synthesis, mediated ketamine's antidepressant activity.

A link between NMDAR and the mTOR pathway was not previously suspected, as the pathway is typically activated by upstream signaling through cell-surface growth factor receptors, not through ligand-gated ion channels like NMDAR.

In normal rats, a single low dose of ketamine rapidly activated mtor signaling in the prefrontal cortex compared with vehicle control $(p<0.01)$. Moreover, ketamine-induced mtor activation correlated with rapid increases in levels of at least three synapse-associated proteins and greater formation of new synapses ( $p<0.01$ for both) compared with what was seen using vehicle.

The same ketamine dose produced a rapid antidepressant effect in two rodent behavioral models of depression and in a rodent model of anxiety. Data were published in Science.

The research "highlights a new path for rapid-onset antidepressant drug development that might bypass NMDA receptor blockade and therefore avoid the undesired cognitive and perceptual changes as well as the potential addiction risk associated with ketamine," John Krystal told SciBX.

Krystal, a professor of translational research and chair of the Department of Psychiatry at the Yale medical school, was a principal investigator on the 2000 ketamine depression trial.

\section{Sticking with NMDAR}

Despite the new findings, Carlos Zarate, a principal investigator on the 2006 ketamine depression trial, still favors looking for safer ways of directly targeting NMDAR. "If we stick with targeting the NMDA receptor rather than go downstream, we probably have two therapeutic options in depression: refine the way we use ketamine in the clinic or use a different class of NMDA receptor antagonist altogether," he told SciBX.

Last month, Zarate's team published a paper in Archives of General Psychiatry showing that a single dose of ketamine reduced bipolar depression in $71 \%$ of treatment-resistant patients compared with placebo, which reduced bipolar depression in only $6 \%$ of patients. ${ }^{11}$

"It may be possible to avoid ketamine's undesirable side effects by combining it with a second compound that counteracts its propsychotic effects", said Zarate. "There's also the possibility of developing a delivery route or vehicle that gets the right dose of ketamine to the right regions of the brain to treat depression without causing psychosis."

Zarate, who is chief of the Experimental Therapeutics \& Pathophysiology Branch in the Division of Intramural Research at the NIMH, and 
Krystal are authors on a 2007 U.S. patent entitled, "Intranasal administration of ketamine to treat depression," which is held jointly by the U.S. government, Yale University and the Mount Sinai School of Medicine. Another author on the patent, Husseini Manji, is now global therapeutic area head of neuroscience R\&D at Johnson \& Johnson.

Zarate also liked that the Yale team's Science paper looked at classes of molecules that antagonize NMDAR differently than ketamine. Indeed, a selective antagonist of the NMDA receptor NR2B subtype (GRIN2B; NR2B) activated mTOR signaling, increased levels of synapse-associated proteins and produced a rapid antidepressant effect in two rodent depression models.

At least two programs are ongoing to develop subunit-specific NMDAR antagonists in depression. Evotec AG and Roche have EVS101 and EVS103, orally active small molecule antagonists of the NR2B subunit, which are in Phase II and Phase I testing, respectively, for treatment-resistant depression. Earlier this year NeurOp Corp. and Bristol-Myers Squibb Co. partnered to discover and develop NR2B antagonists to treat depression.

Despite the suggestion that a subunit-selective NMDAR antagonist could have antidepressant effects comparable to those of ketamine, $\mathrm{M}$. Margarita Behrens, staff scientist at the Salk Institute, thinks repeat or long-term dosing of any NMDAR antagonist could lead to serious, irreversible psychiatric side effects. The reason, she said, is that NMDAR is a key neurotransmitter receptor expressed throughout the brain.

"One way of looking at a single dose of ketamine to treat depression is that by acutely inducing psychotic-like symptoms in a heavily depressed patient, we're enhancing the excitatory tone of certain regions of their brain. That can have the net effect of getting the depressed patient back to normal levels of cortical activity," said Behrens.

"However," she added, "if repeated chronic dosing of ketamine is necessary, we run the real risk of inducing persistent psychotic states in depressed patients. At that point we're basically treating depression by replacing it with another disease."

Thus, Behrens sees a place for NMDAR antagonists only in acute depression. "In an emergency setting where severe depression is driving the patient to serious suicidal thought and behavior, a single dose of ketamine could be a valuable way to rapidly normalize the patient before putting them on more conventional antidepressants," she said.

\section{Box 1. NOX2-driven schizophrenia.}

A team of European researchers has shown that genetic knockout of the NADPH oxidase 2 (NOX2) enzyme alleviated schizophrenia-like symptoms associated with acute ketamine exposure in mice. ${ }^{13}$ Although ongoing studies will attempt to elucidate the mechanism behind the effect, Swiss biotech GenKyoTex S.A. has IP covering multiple NADPH oxidase (NOX) inhibitors that it believes could yield a NOX2 antagonist to treat schizophrenia.

The five isoforms of NOX are responsible for generating superoxides and reactive oxygen species (ROS). Abnormally high levels of ROS lead to cellular oxidative stress, which is thought to contribute to the aging process, neurodegenerative disease and psychiatric disorders. ${ }^{14}$

Previous work by the European researchers and others had shown that in behavioral ${ }^{15}$ and ketamine-induced rodent models ${ }^{16}$ of schizophrenia, the Nox2 enzyme is abnormally overexpressed, leading to downstream oxidative stress, neuronal dysfunction and behavioral abnormalities.

Those findings suggested that selectively blocking NOX2 could reduce oxidative stress, improve neuronal function and reduce schizophrenia symptoms.

To test that hypothesis, the team of researchers on the current study, led by Karl-Heinz Krause, professor of medicine at the Geneva University Hospitals, performed genetic knockout of Nox2 on mouse models of ketamine-induced schizophrenia.

They found the knockout significantly protected the mice from ketamine-induced behavioral abnormalities compared with wild-type mice $(p<0.001)$ and did so, at least partly, by reducing oxidative stress in the prefrontal cortex.

"We're now undertaking studies of postmortem samples from schizophrenia patients to look for markers of NOX2 expression and ROS production in various regions of the brain," said corresponding author Krause.

He told SciBX that the researchers also plan to do further mechanistic studies to better understand how NMDA receptor (NMDAR) blockade by ketamine leads to NOX2 activation and oxidative stress in the CNS.

M. Margarita Behrens, staff scientist at the Salk Institute, told SciBX it will also be important to look at the immunological consequences of NOX2 blockade.

"NOX2 is expressed in neutrophils and plays an important role in the innate immune response. Future studies will need to address the possibility that excessive NOX2 blockade could lead to immunological abnormalities," she said. "Ideally, one would like to find a way to block NOX2 in neurons without impairing its function in neutrophils."

Krause is cofounder of GenKyoTex, a biotech that specializes in developing inhibitors of the five NOX isoforms. ${ }^{17}$ Its lead compound is a NOX4 inhibitor in preclinical development to treat pulmonary fibrosis. According to Krause, the company plans to move that compound into a Phase I safety trial by year end.

Although Krause is aware that global NOX2 inhibition could have undesirable immunological side effects, he said the company is developing selective inhibitors for all the isoforms and hopes to eventually study NOX2 inhibitors in psychiatric and neurodegenerative diseases. $-T F$ 
Behrens added that ketamine would need to be contraindicated for depressed children. "The developing brain is less resilient than the adult brain, and even a single dose of ketamine could cause irreversible damage in children," she said.

Zarate said that his ongoing work includes using a multimodal neuroimaging approach to further evaluate how a single dose of ketamine has rapid antidepressant effects in patients with treatment-resistant major depression and bipolar depression. He said he is also studying NMDAR antagonists that selectively target the NR2B subunit.

\section{Getting more from mTOR}

Safety also will be a key consideration for antidepressants that work downstream of NMDAR, such as mTOR activators.

"Pharmacologically enhancing mTOR signaling is expected to promote proliferation and be potentially carcinogenic, especially in a chronic treatment setting," noted Zarate. "Thus, if an mTOR-activating strategy has a chance in depression, it's probably essential that its longterm safety be demonstrated in patients."

Behrens suggested looking for other downstream targets that are upregulated in response to ketamine and mTOR activation. "By looking at the transcriptional effects of long-term NMDA receptor blockade, it may be possible to find downstream targets for therapeutic intervention that are safer than ketamine but nonetheless yield an antidepressant effect," she said.

As project scientist in the lab of Laura Dugan at the University of California, San Diego, Behrens showed that IL- 6 could fit the bill. She reported in The Journal of Neuroscience that the molecule mediated some of ketamine's propsychotic effects in mice. ${ }^{12}$ Behrens said the cytokine also might be important to ketamine's antidepressant effects. Dugan is chief of the Division of Geriatric Medicine at UCSD.

Yale's Duman told SciBX that his lab is now interested in studying how the mTOR pathway interacts with other cellular pathways, such as the MAP kinase (MAPK; ERK)/protein kinase B (PKB; Akt) pathway, in mediating the antidepressant effects of NMDAR blockade.
According to Duman, the findings reported in Science are unpatented.

Fulmer, T. SciBX 3(34); doi:10.1038/scibx.2010.1032

Published online Sept. 2, 2010

\section{REFERENCES}

1. Li, N. et al. Science; published online Aug. 20, 2010; doi:10.1126/science. 1190287

Contact: Ronald S. Duman, Yale School of Medicine, New Haven, Conn. e-mail: ronald.duman@yale.edu

2. Krystal, J.H. et al. Psychopharmacology (Berl) 169, 215-233 (2003)

3. Gordon, J.A. Nat. Neurosci. 13, 2-4 (2010)

4. Paul, I.A. \& Skolnick, P. Ann. N.Y. Acad. Sci. 1003, 250-272 (2003)

5. Pittenger, C. et al. CNS Neurol. Disord. Drug Targets 6, 101-115 (2007)

6. Petrenko, A.B. et al. Anesth. Analg. 97, 1108-1116 (2003)

7. Berman, R.M. et al. Biol. Psychiatry 47, 351-354 (2000)

8. Zarate, C.A. Jr. et al. Arch. Gen. Psychiatry 63, 856-864 (2006)

9. Krishnan, V. \& Nestler, E.J. Nature 455, 894-902 (2008)

10. Sheline, Y.I. Biol. Psychiatry 54, 338-352 (2003)

11. Diazgranados, N. et al. Arch. Gen. Psychiatry 67, 793-802 (2010)

12. Behrens, M.M. et al. J. Neurosci. 28, 13957-13966 (2008)

13. Sorce, S. et al. J. Neurosci.; published online Aug. 25, 2010; doi:10.1523/JNEUROSCI.1491-10.2010

Contact: Karl-Heinz Krause, Geneva University Hospitals, Geneva, Switzerland e-mail: Karl-Heinz.Krause@unige.ch

14. Sorce, S. \& Krause, K.-H. Antioxid. Redox Signal. 11, 2481-2504 (2009)

15. Schiavone, S. et al. Biol. Psychiatry 66, 384-392 (2009)

16. Behrens, M.M. et al. Science 318, 1645-1647 (2007)

17. Jaquet, V. et al. Antioxid. Redox Signal. 11, 2535-2552 (2009)

COMPANIES AND INSTITUTIONS MENTIONED

Bristol-Myers Squibb Co. (NYSE:BMY), New York, N.Y. Evotec AG (Xetra:EVT), Hamburg, Germany Geneva University Hospitals, Geneva, Switzerland GenKyoTex S.A., Geneva, Switzerland Johnson \& Johnson (NYSE:JNJ), New Brunswick, N.J. Mount Sinai School of Medicine, New York, N.Y. National Institute of Mental Health, Bethesda, Md. National Institutes of Health, Bethesda, Md. NeurOp Corp., Atlanta, Ga.

Roche (SIX:ROG; OTCQX:RHHBY), Basel, Switzerland Salk Institute, La Jolla, Calif.

University of California, San Diego, La Jolla, Calif. Yale School of Medicine, New Haven, Conn. 